University of Nebraska - Lincoln

DigitalCommons@University of Nebraska - Lincoln

\title{
Steady-state Green's function solution for moving media with axial conduction
}

\author{
A. Haji-Sheikh \\ University of Texas at Arlington, haji@uta.edu
}

J. V. Beck

Michigan State University

Kevin D. Cole

University of Nebraska-Lincoln, kcole1@unl.edu

Follow this and additional works at: https://digitalcommons.unl.edu/mechengfacpub

Part of the Mechanical Engineering Commons

Haji-Sheikh, A.; Beck, J. V.; and Cole, Kevin D., "Steady-state Green's function solution for moving media with axial conduction" (2010). Mechanical \& Materials Engineering Faculty Publications. 51. https://digitalcommons.unl.edu/mechengfacpub/51

This Article is brought to you for free and open access by the Mechanical \& Materials Engineering, Department of at DigitalCommons@University of Nebraska - Lincoln. It has been accepted for inclusion in Mechanical \& Materials Engineering Faculty Publications by an authorized administrator of DigitalCommons@University of Nebraska Lincoln. 


\title{
Steady-state Green's function solution for moving media with axial conduction
}

\author{
A. Haji-Sheikh \\ Department of Mechanical and Aerospace Engineering, The University of Texas at Arlington, \\ Arlington, TX 76019-0023, USA (Corresponding author: haji@uta.edu ) \\ J. V. Beck \\ Department of Mechanical Engineering, Michigan State University, East Lansing, MI 48824-1226, USA \\ K. D. Cole \\ Department of Mechanical Engineering, University of Nebraska-Lincoln, Lincoln, NE 68588-0656, USA
}

\begin{abstract}
The objective of this presentation is the development of a generalized steady-state Green's function solution to study the temperature field in moving bodies. This type of solution permits the inclusion of different nonhomogeneous boundary conditions, volumetric heat sources, and possible position-dependent thermophysical properties. Although the mathematical formulation is for moving solids, it can be used to study the heat transfer in a moving fluid with a non-uniform velocity distribution passing through a micro-channel or fluid-saturated porous ducts. Additionally, this presentation includes the application of this Green's function solution to acquire numerical information for selected examples to further illustrate the numerical details.
\end{abstract}

Keywords: heat transfer, moving boundary, axial conduction, slug flow, thermal entrance

\section{Introduction}

A detailed description of the Green's function solution for classical thermal conduction applications is given in Beck et al. [1]. Several studies reported in the literature also contain the Green's function solutions to study the heat transfer to moving fluids in different fluid passages. Nagasue [2] presents a Green's function solution in order to study the heat transfer to fluid flowing in circular passages with specified wall temperature or specified wall heat flux. The contribution of axial conduction is included in the study reported in [2]. The contribution of axial conduction in a moving semi-infinite solid is presented in [3] using a step change in the surface temperature and is given in [4] for a step change in the wall heat flux. Tiselj et al. [5] includes the contribution of axial conduction to study heat transfer in micro-channels. In the absence of axial conduction, the Green's function solution is used in [6] to determine the contribution of frictional heating in fluid flowing through triangular passages.

Basic studies related to the contribution of axial conduction in different fluid passages are reported in [7-11]; these studies use the boundary conditions of the first kind with an abrupt change in the wall temperature. The contribution of axial conduction in rectangular passages is studied in [12] using the weighted residual method that is based on variational calculus. The contribution of axial conduction in the presence of the boundary conditions of the second kind is reported in $[13,14]$. When the moving medium is a fluid flowing through a metallic porous passage, the contribution of axial conduc- tion greatly influences the rate of heat transfer. Numerically acquired heat transfer data, in the presence of axial conduction, are presented in [15-17]. An analytical solution that uses an extended Graetz procedure is presented in [18] and an asymptotic solution that provides accurate information near the entrance location is given in [19].

The Green's function for determination of steady-state temperature is available in the literature. Yen and Beck [20] presents a Green's function formulation and solution for three-dimensional steady-state heat-conduction problems in a two-layered body. Cole [21] describes the fin effect in the Green's function solution for temperature and the procedure is extended in Cole [22] for determination of the effect of periodic heating.

The development of a steady-state Green's function solution equation for the energy equation in a moving body, with basic variables in their most general form, is the subject of this presentation. Useful applications include the study of heat transfer to fluid flowing in micro-channels and in fluid-saturated porous ducts. Before developing the Green's function solution for these applications, it is appropriate to itemize certain assumptions to be used in the forthcoming formulation:

1. There is a local thermal equilibrium condition throughout the medium.

2. The velocity of the moving medium, shown in Figure 2, is independent of the axial coordinate $x$, but it can depend on other coordinates, $u=u(y, z)$.

3. There is no mechanical compression of the domain.

4. The thermophysical properties are independent of the axial coordinate. 


\section{Nomenclature}

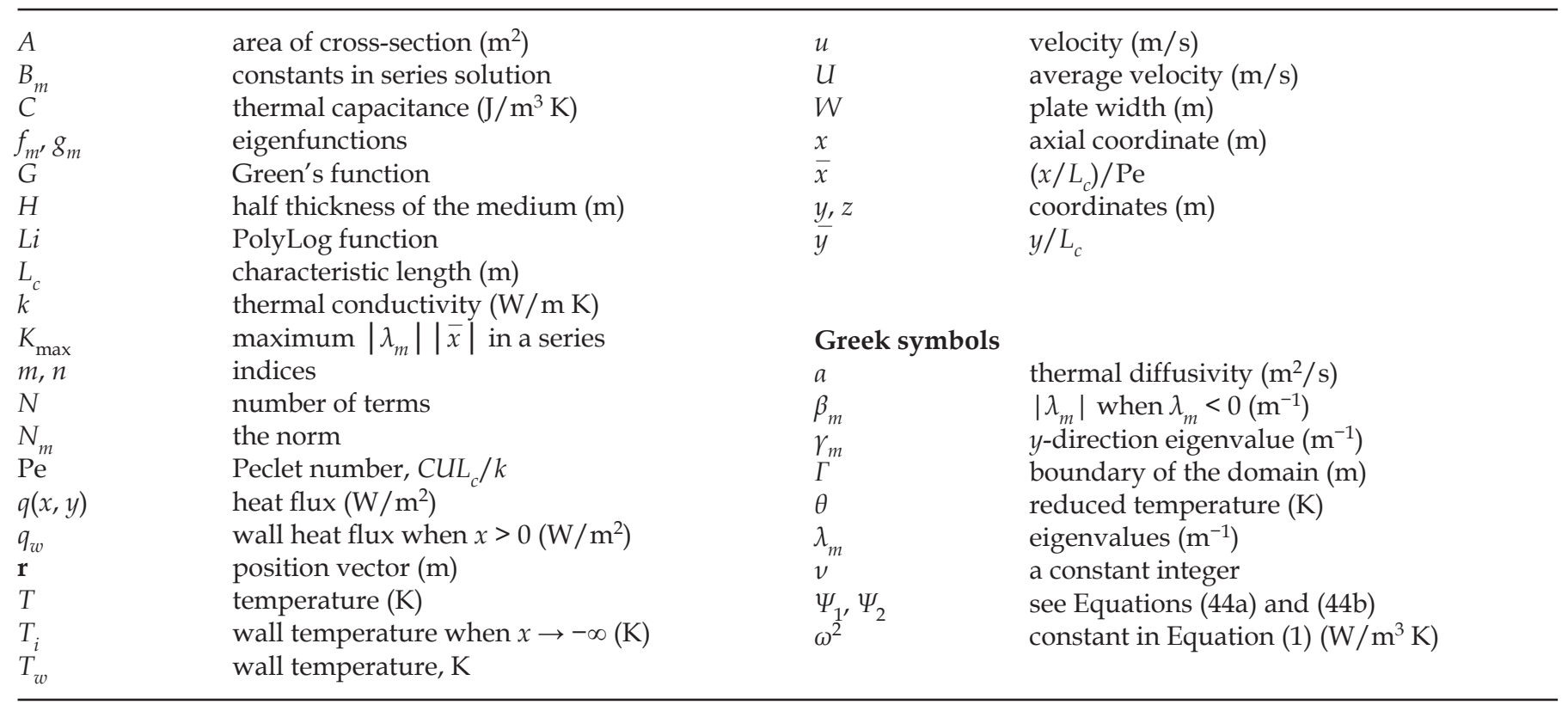

5. The steady-state condition exists throughout the medium.

6. The thermophysical properties are independent of temperature.

\section{Steady-state energy equation}

The mathematical formulation of the steady-state Green's function solution must satisfy the energy equation in its general form,

$$
C u \frac{\partial T}{\partial x}=\frac{\partial}{\partial x}\left(k \frac{\partial T}{\partial x}\right)+\frac{\partial}{\partial y}\left(k \frac{\partial T}{\partial y}\right)+\frac{\partial}{\partial z}\left(k \frac{\partial T}{\partial z}\right)-\omega^{2} T+S(x, y, z)
$$

Here, $S(x, y, z)$ is the volumetric heat source, $C=\rho c_{p}$ is the thermal capacitance, $k$ is the thermal conductivity, and $u$ is the velocity of the moving body. The term $\omega^{2} T$ is often known as the fin effect and it also emerges during steady-periodic heating as described in Cole [23]. Functional dependences are indicated by $C=C(y, z), k=k(y, z)$, and $u=u(y, z)$ while each remains independent of the axial coordinate $x$. Using these specified conditions and for convenience of mathematical formulations, Equation (1) is rewritten as

$$
\frac{\partial}{\partial y}\left(k \frac{\partial T}{\partial y}\right)+\frac{\partial}{\partial z}\left(k \frac{\partial T}{\partial z}\right)-\omega^{2} T+S(x, y, z)=C u \frac{\partial T}{\partial x}-k \frac{\partial^{2} T}{\partial x^{2}}
$$

As a shorthand notation, the first two terms on the left side of Equation (2) are designated as $\nabla \cdot(k \nabla T)$. The second term on the right side of Equation (2) describes the contribution of axial conduction of heat. Then, the more compact form of Equation (2),

$$
\nabla \cdot(k \nabla T)-\omega^{2} T+S(x, y, z)=C u \frac{\partial T}{\partial x}-k \frac{\partial^{2} T}{\partial x^{2}}
$$

is used in the subsequent mathematical formulations.

For a moving solid, the velocity $u$ is a constant equal to $U$; however, $k$ and $C$ could depend on $y$ and $z$. This methodology is equally applicable to a moving fluid when the velocity $u=u(y, z)$ is fully developed within a channel. Before presenting solutions for specific problems, the emphasis of this work is to formulate the Green's function solution and find suitable methods of acquiring the Green's function. Later, this methodology is applied to specific examples to be followed by numerical information.

\section{Green's function solution formulation}

The first task is to establish a definition for the Green's function. The next task is to present the Green's function solution formulation followed by a method for determination of the Green's function.

As shorthand notations, let $\mathbf{r}$ and $\mathbf{r}^{\prime}$ be the position vectors within the medium's $y$-z-plane and let $G$ stand for the Green's function $G\left(x, \mathbf{r} \mid \xi, \mathbf{r}^{\prime}\right)$. Here, both $x$ and $\xi$ represent the axial coordinate while $\mathbf{r}$ stands for the $(y, z)$ coordinates. As usual, the Green's function describes the temperature at point $(x, \mathbf{r})$ due to an energy pulse that occurs at the $\left(\xi, \mathbf{r}^{\prime}\right)$ location. Now, it is appropriate to formulate a methodology for finding the thermal response at location $(x, \mathbf{r})$ when there is a source located at $\left(\xi, \mathbf{r}^{\prime}\right)$.

For a domain depicted in Figure 1, the Green's function relation that includes the contribution of an energy source $C \delta\left(\mathbf{r}-\mathbf{r}^{\prime}\right) \delta(x-\xi)$ is the partial differential equation

$$
\nabla \cdot(k \nabla G)-\omega^{2} G+C \delta\left(\mathbf{r}-\mathbf{r}^{\prime}\right) \delta(x-\xi)=C u \frac{\partial G}{\partial x}-k \frac{\partial^{2} G}{\partial x^{2}}
$$

wherein $\delta$ represents the Dirac delta function. In this definition the Green's function has units of $\mathrm{s} / \mathrm{m}^{3}$. The Green's functions for different bodies are commonly obtained with specified homogeneous boundary conditions. Then, using the acquired Green's function, one can use the Green's function solution to get numerical information for related problems with non-homogeneous boundary conditions.

The process of developing the Green's function solution begins by exchanging the spatial variables $\mathbf{r}$ and $\mathbf{r}^{\prime}$ in Equation (4)

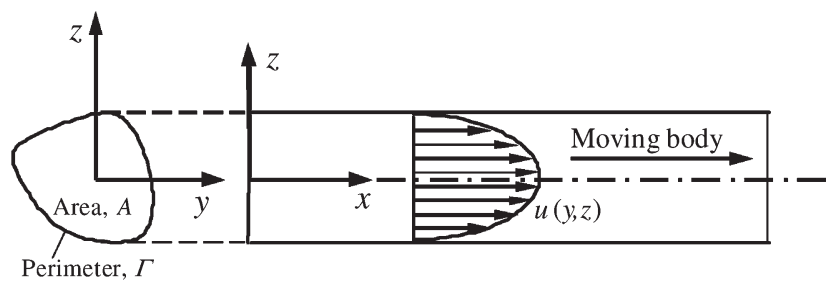

Figure 1. Schematic of a moving domain and the coordinate system. 
and changing the axial coordinate $x$ to $\xi$ and $\xi$ to $x$. Then, after appropriate substitutions, Equation (4) becomes

$$
\nabla_{0} \cdot\left(k \nabla_{0} G\right)-\omega^{2} G+C \delta\left(\mathbf{r}^{\prime}-\mathbf{r}\right) \delta(x-\xi)=-C u \frac{\partial G}{\partial \xi}-k \frac{\partial^{2} G}{\partial \xi^{2}}
$$

where $\nabla_{o}$ is now the "grad" operator in the $\mathbf{r}^{\prime}$ space. Moreover, the diffusion equation, Equation (3), in ( $\left(\mathbf{r}^{\prime} \mathbf{r}^{\prime}\right)$ space takes the form

$$
\nabla_{0} \cdot\left(k \nabla_{0} T\right)-\omega^{2} T+S\left(\xi, \mathbf{r}^{\prime}\right)=C u \frac{\partial T}{\partial \xi}-k \frac{\partial^{2} T}{\partial \xi^{2}}
$$

Now, multiplying Equation (5) by $-T$ and Equation (6) by $G$ and then adding the resulting relations produces the equation

$$
\begin{aligned}
& =C u \frac{\partial(T G)}{\partial \xi}-k\left(\frac{\partial^{2} T}{\partial \xi^{2}} G-\frac{\partial^{2} G}{\partial \xi^{2}} T\right) \\
& =C u \frac{\partial(T G)}{\partial \xi}-k\left[\frac{\partial}{\partial \xi}\left(G \frac{\partial T}{\partial \xi}\right)-\frac{\partial}{\partial \xi}\left(T \frac{\partial G}{\partial \xi}\right)\right]
\end{aligned}
$$$$
G \nabla_{0} \cdot\left(k \nabla_{0} T\right)-T \nabla_{0} \cdot\left(k \nabla_{0} G\right)+G S\left(\mathbf{r}^{\prime}, \xi\right)-C \delta\left(\mathbf{r}^{\prime}-\mathbf{r}\right) \delta(x-\xi) T
$$

Using standard procedures in [1, Section 10.2.6], both sides of Equation (7) are integrated over cross-sectional area $A$ with $\mathbf{r}^{\prime}$ representing the spatial variables and integrated over $\xi$ from $\xi=\xi_{0}$ to $\infty$. Then, using the application of the Green's theorem, one gets the desired form of the steady-state Green's function solution equation for axial flow in $x$-direction

$$
\begin{aligned}
T(x, \mathbf{r})= & \frac{1}{C(\mathbf{r})}\left\{\int_{\xi=\xi}^{\infty} d \xi \int_{\Gamma} k\left(\mathbf{r}^{\prime}\right)\left(G \frac{\partial T}{\partial n}-T \frac{\partial G}{\partial n}\right)_{\Gamma^{\prime}} d \Gamma^{\prime}\right. \\
& +\int_{\xi=\xi_{0}}^{\infty} d \xi \int_{A} G S\left(\xi, \mathbf{r}^{\prime}\right) d A^{\prime}+\left.\int_{A} C\left(\mathbf{r}^{\prime}\right) u\left(\mathbf{r}^{\prime}\right) G\right|_{\xi=\xi_{0}} T\left(\mathbf{r}^{\prime}, \xi_{0}\right) d A^{\prime} \\
& \left.-\int_{A} k\left(\mathbf{r}^{\prime}\right)\left[G \frac{\partial T\left(\mathbf{r}^{\prime}, \xi\right)}{\partial \xi}\right]_{\xi=\xi_{0}} d A^{\prime}+\int_{A} k\left(\mathbf{r}^{\prime}\right)\left[\frac{\partial G}{\partial \xi} T\left(\xi, \mathbf{r}^{\prime}\right)\right]_{\xi=\xi_{0}} d A^{\prime}\right\}
\end{aligned}
$$

where $A$ stands for the cross-section of the domain in $y-z$ plane and $\Gamma$ is its perimeter, see Figure 1 . It is to be noted that both $\left.G\right|_{\xi \rightarrow \infty}$ and $\partial G /\left.\partial \xi\right|_{\xi \rightarrow \infty}$ vanish, as $\xi \rightarrow \infty$. On the right side of Equation (8), there are three contributions within Equation (8): (1) the boundary condition effects are in the first term, (2) the volumetric heat source effect is in the second term, and (3) the last three terms describe the effects due to the entrance conditions.

Once the Green's function is known, Equation (8) can provide the temperature solution within the material domain. The determinations of the Green's function for a few special cases are the considered next. Different boundary conditions are considered in the forthcoming formulations; e.g., the first kind for prescribed surface temperature and the second kind for prescribed surface heat flux.

\section{Green's function determination}

The method of Green's function determination depends on the specific geometry. Several approaches are available for stationary solids with one given in [20]. Reference [20]provides a method for deriving Green's function with other convergence characteristics. A standard practice for determination of the Green's function is to acquire a temperature solution hypothesizing that the conditions $T(0, y, z)$ and $\partial T(x, y, z) /\left.\partial x\right|_{x=0}$ are prescribed, in the presence of homogeneous boundary conditions. Then, the Green's function is obtainable by comparing this temperature solution with the corresponding terms in Equation (8). Herein, consideration is given to the moving bodies having uniform cross-section profiles. For this application, a solution commonly has the form of an infinite series. Then, in the absence of a volumetric heat source and for homogeneous boundary conditions of the 1st, 2nd, and 3rd kind, as described in [1, Chapter 10], one can consider a temperature solution in its basic dimensional form as

$$
T(x, y, z)=\sum_{m=1}^{\infty} B_{m} f_{m}(y, z) e^{-\lambda_{m} x}
$$

The form of this equation ensures exponential convergence provided $x$ is not equal to zero. Also, it is necessary to find the derivative of $T(x, y, z)$ as

$$
\frac{\partial T(x, y, z)}{\partial x}=-\sum_{m=1}^{\infty} B_{m} \lambda_{m} f_{m}(y, z) e^{-\lambda_{m} x}
$$

For determination of $B_{m^{\prime}}$ these two relations, Equations (9) and (10)(9) and (10), at $x=0$ location become

$$
\begin{aligned}
& T(0, y, z)=\sum_{m=1}^{\infty} B_{m} f_{m}(y, z) \\
& \left.\frac{\partial T(x, y, z)}{\partial x}\right|_{x=0}=-\sum_{m=1}^{\infty} B_{m} \lambda_{m} f_{m}(y, z)
\end{aligned}
$$

The orthogonality condition, Equation (A.9a), in Appendix A, suggests multiplying both sides of the first equation by $\left(k \lambda_{n}+C u\right) f_{n}(y, z) d y d z$, both sides of the second equation by $-k f_{n}(y, z) d y d z$, and then adding the resulting relations to get

$$
\begin{aligned}
& \left(k \lambda_{n}+C u\right) T(0, y, z) f_{n}(y, z) d y d z \\
& \quad-\left.k[\partial T(x, y, z) / \partial x]\right|_{x=0} f_{n}(y, z) d y d z \\
& \quad=\sum_{m=1}^{\infty} B_{m}\left[k\left(\lambda_{m}+\lambda_{n}\right)+C u\right] f_{m}(y, z) f_{n}(y, z) d y d z
\end{aligned}
$$

Next, for a specified index $n$, one can integrate both sides of this equation over the cross-sectional area and then use the orthogonality condition in Appendix A to get

$$
\begin{aligned}
B_{n}= & \frac{1}{N_{n}} \int_{A}\left(k \lambda_{n}+C u\right) T(0, y, z) f_{n}(y, z) d A-\frac{1}{N_{n}} \\
& \times\left.\int_{A} k[\partial T(x, y, z) / \partial x]\right|_{x=0} f_{n}(y, z) d A
\end{aligned}
$$

where

$$
N_{n}=\int_{A}\left(2 k \lambda_{n}+C u\right)\left[f_{n}(y, z)\right]^{2} d A
$$

where $d A=d y d z$ and $m=n$ in this orthogonality process. The parameters $x, y, z$ in this equation are dummy variables and they are replaced by $\xi, y^{\prime}$, and $z^{\prime}$ in the next relation. Then, after substitution for $B_{m}$ from Equation (13a) in Equation (9), a reduced form of Equation (9) is

$$
\begin{aligned}
T(x, y, z)= & \sum_{m=1}^{\infty} \frac{1}{N_{m}} e^{-\lambda_{m} x} \int_{A} \operatorname{CuT}\left(0, y^{\prime}, z^{\prime}\right) f_{m}(y, z) f_{m}\left(y^{\prime}, z^{\prime}\right) d A^{\prime} \\
& +\sum_{m=1}^{\infty} \frac{\lambda_{m}}{N_{m}} e^{-\lambda_{m} x} \int_{A} k T\left(0, y^{\prime}, z^{\prime}\right) f_{m}(y, z) f_{m}\left(y^{\prime}, z^{\prime}\right) d A^{\prime} \\
& -\sum_{m=1}^{\infty} \frac{1}{N_{m}} e^{-\lambda_{m} x} \\
& \times \int_{A} k\left(\frac{\partial T\left(\xi, y^{\prime}, z^{\prime}\right)}{\partial \xi}\right)_{\xi=0} f_{m}(y, z) f_{m}\left(y^{\prime}, z^{\prime}\right) d A^{\prime}
\end{aligned}
$$

where $d A^{\prime}=d y^{\prime} d z^{\prime}$. This equation also yields the temperature distribution when $x<0$ by using the negative $\lambda_{m}$ values. By selecting $x$ to have a negative value, it introduces a negative sign before velocity $u$, replaces $\lambda_{m}$ with $-\beta_{m^{\prime}}$ and it will add a negative sign to the last term because $\xi$-direction changes as $x$-direction changes.

The temperature solution as given by Equation (14) is now compared to the Green's function solution equation as given by Equation (8) when $\xi_{o}=0$. Then, for a domain having homo- 
geneous boundary condition and without any volumetric heat source, Equation (8) keeping only the last three terms with basic variables becomes

$$
\begin{aligned}
T(x, y, z)= & \frac{1}{C(y, z)}\left\{\int_{A} C\left(y^{\prime}, z^{\prime}\right) u\left(y^{\prime}, z^{\prime}\right) G_{\xi=0} T\left(y^{\prime}, z^{\prime}, \xi_{0}\right) d A^{\prime}\right. \\
& +\int_{A} k\left(y^{\prime}, z^{\prime}\right)\left[\frac{\partial G}{\partial \xi} T\left(y^{\prime}, z^{\prime}, \xi\right)\right]_{\xi=0} d A^{\prime}-\int_{A} k\left(y^{\prime}, z^{\prime}\right) \\
& \left.\times\left[G \frac{\partial T\left(y^{\prime}, z^{\prime}, \xi\right)}{\partial \xi}\right]_{\xi=0} d A^{\prime}\right\}
\end{aligned}
$$

Since the coefficient $C(y, z)$ on the right side of Equation (15) is independent of $y^{\prime}$ and $z^{\prime}$, it can be moved inside of the integrals and combined with the function $G=G\left(x, y, z \mid \xi, y^{\prime}, z^{\prime}\right)$ Accordingly, Equations (14) and (15)(14) and (15) become the same when $G / C(y, z)$ takes the form

$$
\frac{G\left(x, y, z \mid \xi, y^{\prime}, z^{\prime}\right)}{C(y, z)}=\sum_{m=1}^{\infty} \frac{f_{m}(y, z) f_{m}\left(y^{\prime}, z^{\prime}\right)}{N_{m}} e^{-\lambda_{m}(x-\xi)}
$$

wherein the norm $N_{m^{\prime}}$ as given in Appendix A, is

$$
N_{m}=\int_{A}\left[2 \lambda_{m} k+C\left(y^{\prime}, z^{\prime}\right) u\left(y^{\prime}, z^{\prime}\right)\right]\left[f_{m}\left(y^{\prime}, z^{\prime}\right)\right]^{2} d A
$$

It is to be noted that this Green's function relation is applicable to the case when an energy pulse appears at $x=\xi$ location and its effect is sought at other locations when $x-\xi>0$. Also, as stated earlier, this Green's function relation is applicable to the case when $x-\xi<0$ after replacing $\lambda_{m}$ with $-\beta_{m}$ in Equations (16a) and (16b) and changing $N_{m}$ in Equation (16a) with $N_{m, 1}$ that becomes $-N_{m}$. This formulation implies that this Green's function solution with the Green's functions as defined in Equation (16a) is applicable to a moving body having boundary conditions of the first kind, second kind, or third kind, once appropriate eigenfunctions and corresponding eigenvalues are determined for that specific moving body. Therefore, this methodology is applicable to the study of temperature field in moving solids and to moving fluids in different passages including micro-channels.

\section{Effect of boundary conditions}

The Green's function solution, as given by Equation (8), is applicable using various non-homogeneous boundary conditions. However, two types of boundary conditions are being considered for this presentation: the first kind and the second kind.

\subsection{Boundary conditions of the first kind}

As a test case, for verification of the aforementioned procedure, consider a constant capacitance $C$ and a constant thermal conductivity $k$. Also, assume this moving body has a uniform temperature equal to $T_{i}$ when $x=-\infty$. It is appropriate to use a reduced temperature $T-T_{i}$ with its derivative $\partial T / \partial \xi=0$ at $x=-\infty$. Furthermore, for the boundary conditions of the first kind, the Green's function $G=0$ at the boundary and then a reduced form of Equation (8) when $\xi_{o}=-\infty$ and $S(x, y, z)=0$ is

$$
T(x, y, z)-T_{i}=-\frac{k}{C} \int_{\xi=-\infty}^{\infty} d \xi \int_{\Gamma}\left(\left(T_{w}(\xi)-T_{i}\right) \frac{\partial G}{\partial n}\right)_{\Gamma^{\prime}} d \Gamma^{\prime}
$$

The wall temperature $T_{w}(x)$ in Equation (17) is selected to be independent of $y$ and $z$. For this case, the Green's function has two distinct forms, which are:

$$
G_{1}\left(x, y, z \mid \xi, y^{\prime}, z^{\prime}\right)=\sum_{m=1}^{\infty}\left(\frac{C}{N_{m}}\right) f_{m}(y, z) f_{m}\left(y^{\prime}, z^{\prime}\right) e^{\mid \lambda_{m}(x-\xi)}
$$

when $\lambda_{m}<0$ for $\xi>x$ and

$$
G_{2}\left(y, z, x \mid y^{\prime}, z^{\prime}, \xi\right)=\sum_{m=1}^{\infty}\left(\frac{C}{N_{m}}\right) f_{m}(y, z) f_{m}\left(y^{\prime}, z^{\prime}\right) e^{-\lambda_{m}(x-\xi)}
$$

when $\lambda_{m} \geq 0$ for $\xi<x$. When $\lambda_{m}<0$, let $\beta_{m}=\left|\lambda_{m}\right|$, $g_{m}(y, z)=f_{m}(y, z)$, and $N_{m, 1}=N_{m}$ in the below relations, for convenience of mathematical formulation. Then, the substitution of the Green's function from Equations (18a) and (18b) into Equation (17) produces the temperature solution as

$$
\begin{aligned}
T(x, y, z)-T_{i}= & -k\left\{\sum_{m=1}^{\infty} \frac{f_{m}(y, z)}{N_{m}} \int_{\xi=-\infty}^{x}\left[T_{w}(\xi)-T_{i}\right] e^{-\lambda_{m}(x-\xi)} d \xi\right. \\
& \times \int_{\Gamma}\left(\frac{\partial f_{m}\left(y^{\prime}, z^{\prime}\right)}{\partial n}\right)_{\Gamma^{\prime}} d \Gamma^{\prime}+\sum_{m=1}^{\infty} \frac{g_{m}(y, z)}{N_{m, 1}} \int_{\xi=x}^{\infty}\left[T_{w}(\xi)-T_{i}\right] \\
& \left.\times e^{\beta_{m}(x-\xi)} d \xi \int_{\Gamma}\left(\frac{\partial g_{m} y^{\prime}, z^{\prime}}{\partial n}\right)_{\Gamma^{\prime}} d \Gamma^{\prime}\right\}
\end{aligned}
$$

Furthermore, let $T_{w}(x)=T_{i}$ when $x<0$ and $T_{w}(x)=T_{w}$, as a constant, when $x \geq 0$ and then, the above equation, when $x<0$, reduces to

$$
\begin{aligned}
T(x, y, z)-T_{i}= & -\sum_{m=1}^{\infty} \int_{\xi=0}^{\infty} k\left[T_{w}-T_{i}\right] \\
& \times \frac{g_{m}(y, z)}{N_{m, 1}} e^{\beta_{m}(x-\xi)} d \xi \int_{\Gamma}\left(\frac{\partial g_{m}\left(y^{\prime}, z^{\prime}\right)}{\partial n}\right)_{\Gamma^{\prime}} d \Gamma^{\prime}
\end{aligned}
$$

while when $x>0$, the solution has the form

$$
\begin{aligned}
T(x, y, z)-T_{i}= & -\left\{\sum_{m=1}^{\infty} \frac{f_{m}(y, z)}{N_{m}} \int_{\xi=0}^{x} k\left(T_{w}-T_{i}\right) e^{-\lambda_{m}(x-\xi)} d \xi\right. \\
& \times \int_{\Gamma}\left(\frac{\partial f_{m}\left(y^{\prime}, z^{\prime}\right)}{\partial n}\right)_{\Gamma^{\prime}} d \Gamma^{\prime}+\sum_{m=1}^{\infty} \frac{g_{m}(y, z)}{N_{m, 1}} \int_{\xi=x}^{\infty} k\left(T_{w}-T_{i}\right) \\
& \left.\times e^{\beta_{m}(x-\xi)} d \xi \int_{\Gamma}\left(\frac{\partial g_{m}\left(y^{\prime}, z^{\prime}\right)}{\partial n}\right)_{\Gamma^{\prime}} d \Gamma^{\prime}\right\}
\end{aligned}
$$

To compare Equations (20) and (21)(20) and (21) with the existing solutions given in [18], the contour integrals can be converted to volume integrals. To perform this task, one can substitute $f_{m}(y, z) \exp \left(-\lambda_{m} x\right)$ from Equation (9) into Equation (3) with $\omega^{2}=0$ and $S(x, y, z)=0$ to get the relation

$$
\nabla \cdot\left[k \nabla f_{m}(y, z)\right]=-C u \lambda_{m} f_{m}(y, z)-k \lambda_{m}^{2} f_{m}(y, z)
$$

The integration of Equation (22) over the cross-sectional area $A$ and after using the divergence theorem provides the relation

$$
k \int_{\Gamma}\left(\frac{\partial f_{m}\left(y^{\prime}, z^{\prime}\right)}{\partial n}\right)_{\Gamma^{\prime}} d \Gamma^{\prime}=-\int_{A}\left(C u \lambda_{m}+k \lambda_{m}^{2}\right) f_{m}\left(y^{\prime}, z^{\prime}\right) d A^{\prime}
$$

for positive and negative values of $\lambda_{m}$. To verify the accuracy of this solution obtained by the application of the Greens function, it is possible to acquire a solution for this special case by an existing procedure presented in $[9,10,18]$. For this moving body, it is customary to define two reduced solution functions

$$
\theta_{1}(x, y, z)=T(x, y, z)-T_{i} \quad \text { when } x<0
$$

and

$$
\theta_{2}(x, y, z)=T(x, y, z)-T_{w} \text { when } x>0
$$

The first function $\theta_{1}(x, y, z)$ is the solution of the energy equation assuming $\theta_{1}=0$ at $x=-\infty$ while, at the wall, $\theta_{1}=0$ when $x<0$. Next, the second function $\theta_{2}(x, y, z)$ is for the $x \geq 0$ condition. It is to be noted that at $x=0$, the continuity of temperature $T(x, y, z)$ requires the condition that

$$
\theta_{2}(0, y, z)=\theta_{1}(0, y, z)-\left(T_{w}-T_{i}\right)
$$

while the continuity of heat flux at $x=0$ makes

$$
\left[\frac{\partial \theta_{2}(x, y, z)}{\partial x}\right]_{x=0}=\left[\frac{\partial \theta_{1}(x, y, z)}{\partial x}\right]_{x=0}
$$


Then, these two compatibility conditions in conjunction with the orthogonality condition from Equation (A.6), when $\lambda_{m}<0$ for $x<0$, would provide the solution for $\theta_{1}$, using Equation (A.1),

$$
\theta_{1}=\sum_{m=1}^{\infty}\left(T_{w}-T_{i}\right) \frac{\int_{A}\left(k \beta_{m}-C u\right) g_{m}(y, z) g_{m}\left(y^{\prime}, z^{\prime}\right) d A^{\prime}}{\int_{A}\left(2 k \beta_{m}-C u\right)\left[g_{m}\left(y^{\prime}, z^{\prime}\right)\right]^{2} d A^{\prime}} e^{\beta_{m} x}
$$

Next, the Green's function solution, after using Equations (20) and (23), produces the relation

$$
\begin{aligned}
\theta_{1} & =\sum_{m=1}^{\infty} \int_{\xi=0}^{\infty}\left(T_{w}-T_{i}\right) \frac{g_{m}(y, z)}{N_{m, 1}} e^{\beta_{m}(x-\xi)} d \xi \beta_{m} \int_{A}\left(C u-k \beta_{m}\right) g_{m}\left(y^{\prime}, z^{\prime}\right) d A^{\prime} \\
& =\sum_{m=1}^{\infty}\left(T_{w}-T_{i}\right) \frac{\int_{A}\left(C u-k \beta_{m}\right) g_{m}(y, z) g_{m}\left(y^{\prime}, z^{\prime}\right) d A^{\prime}}{\int_{A}\left(C u-2 k \beta_{m}\right)\left[g_{m}\left(y^{\prime}, z^{\prime}\right)\right]^{2} d A^{\prime}} e^{\beta_{m} x}
\end{aligned}
$$

which is the same as the existing solution given by Equation (26). The denominator in this equation is designated as $N_{m 1}$ and it is the norm obtainable from Equation (A.9b) of Appen$\operatorname{dix}$ A when $\beta_{m}=-\lambda_{m}$ while $\lambda_{m}<0$.

Repeating the process that produced Equation (26) for the case when $\lambda_{m}>0$ for $x>0$, would provide the solution for $\theta_{2}(x, y, z)$ function and then the temperature solution takes the form

$$
\begin{aligned}
\frac{T(x, y, z)-T_{i}}{T_{w}-T_{i}} & =1+\frac{\theta_{2}(x, y, z)}{T_{w}-T_{i}} \\
& =1-\sum_{m=1}^{\infty} \frac{\int_{A}\left(k \lambda_{m}+C u\right) f_{m}(y, z) f_{m}\left(y^{\prime}, z^{\prime}\right) d A^{\prime}}{\int_{A}\left(2 k \lambda_{m}+C u\right)\left[f_{m}\left(y^{\prime}, z^{\prime}\right)\right]^{2} d A^{\prime}} e^{-\lambda_{m} x}
\end{aligned}
$$

since

$$
\frac{T(x, y, z)-T_{i}}{T_{w}-T_{i}}-\frac{T(x, y, z)-T_{w}}{T_{w}-T_{i}}=1
$$

The denominator in this equation is designated as $N_{m}$, as given by Equation (A.9b) of Appendix A. The Green's function solution after combining Equation (21) and Equation (23) becomes

$$
\begin{aligned}
\frac{T(y, z, x)-T_{i}}{\left(T_{w}-T_{i}\right)}= & \sum_{m=1}^{\infty} \frac{f_{m}(y, z)}{N_{m}} e^{-\lambda_{m} x} \int_{\xi=0}^{x} e^{\lambda_{m} \xi} d \xi \\
& \times \int_{A}\left(C u \lambda_{m}+k \lambda_{m}^{2}\right) f_{m}\left(y^{\prime}, z^{\prime}\right) d A^{\prime}+\sum_{m=1}^{\infty} \frac{g_{m}(y, z)}{N_{m, 1}} e^{\beta_{m} x} \\
& \times \int_{\xi=x}^{\infty} e^{-\beta_{m} \xi} d \xi \int_{A}\left(C u \beta_{m}-k \beta_{m}^{2}\right) g_{m}\left(y^{\prime}, z^{\prime}\right) d A^{\prime}
\end{aligned}
$$

Next, the integral over $\xi$ is evaluated to get

$$
\begin{aligned}
\frac{T(y, z, x)-T_{i}}{\left(T_{w}-T_{i}\right)}= & \sum_{m=1}^{\infty} \frac{f_{m}(y, z)}{N_{m}}\left(1-e^{-\lambda_{m} x}\right) \int_{A}\left(u+k \lambda_{m} / C\right) f_{m}\left(y^{\prime}, z^{\prime}\right) d A^{\prime} \\
& +\sum_{m=1}^{\infty} \frac{g_{m}(y, z)}{N_{m, 1}} \int_{A}\left(u-k \beta_{m} / C\right) g_{m}\left(y^{\prime}, z^{\prime}\right) d A^{\prime}
\end{aligned}
$$

that can be written as

$$
\begin{aligned}
\frac{T(y, z, x)-T_{i}}{\left(T_{w}-T_{i}\right)}= & \sum_{m=1}^{\infty} \frac{g_{m}(y, z)}{N_{m, 1}} \int_{A}\left(C u-k \beta_{m}\right) g_{m}\left(y^{\prime}, z^{\prime}\right) d A^{\prime} \\
& +\sum_{m=1}^{\infty} \frac{f_{m}(y, z)}{N_{m}} \int_{A}\left(C u+k \lambda_{m}\right) f_{m}\left(y^{\prime}, z^{\prime}\right) d A^{\prime} \\
& -\sum_{m=1}^{\infty} \frac{f_{m}(y, z)}{N_{m}} e^{-\lambda_{m} x} \int_{A}\left(C u+k \lambda_{m}\right) f_{m}\left(y^{\prime}, z^{\prime}\right) d A^{\prime}
\end{aligned}
$$

By placing Equations (27) and (28) into Equation (25a), it is to be noted that the sum of the first two terms on the right side of Equation (30) is equal to 1 which is also the limiting condition as $x \rightarrow \infty$; therefore, Equation (30) is the same as Equation (28)

\subsection{Boundary conditions of the second kind}

The procedure in previous section for boundary conditions of the first kind can be modified to get a solution when the boundary condition is of the second kind. As in the previous case, $S(x, y, z)=0$ and the entrance condition is at a $\xi_{0}=-\infty$ location. When the input heat flux is $k \partial T / \partial n$ at the wall, the temperature solution is obtainable from the Green's function contribution as given by the first term in Equation (8); that is

$$
\begin{aligned}
T(x, y, z)-T_{i} & =\frac{1}{C(\vec{r})} \int_{\xi=-\infty}^{\infty} d \xi \int_{\Gamma} k\left(r^{\prime}\right)\left(G \frac{\partial T}{\partial n}\right)_{\Gamma^{\prime}} d \Gamma^{\prime} \\
& =\frac{1}{C(\vec{r})} \int_{\xi=-\infty}^{\infty} d \xi \int_{\Gamma}[q G]_{\Gamma^{\prime}} d \Gamma^{\prime}
\end{aligned}
$$

when $q=k \partial \mathrm{T} / \partial n$ is the heat flux entering the body. The functional form of the Green's function is given by Equations (18a) and $(18 \mathrm{~b})$ with appropriate eigenfunctions and eigenvalues. The wall heat flux in Equation (31) can depend $x, y$, and $z$. When the wall heat flux depends only on the axial coordinate, $q=q_{w}(x)$, the temperature solution becomes

$$
\begin{aligned}
T(x, y, z)-T_{i}= & \sum_{m=1}^{\infty} \int_{\xi=-\infty}^{\infty} q_{w}(\xi) \\
& \times \frac{f_{m}(y, z)}{N_{m}} e^{-\lambda_{m}(x-\xi)} d \xi \int_{\Gamma}\left[f_{m}\left(y^{\prime}, z^{\prime}\right)\right]_{\Gamma^{\prime}} d \Gamma^{\prime}
\end{aligned}
$$

wherein $\lambda_{m}$ can be positive or negative. Then, the general solution when $\lambda_{m} \leq 0$ for $\xi>x$ and when $\lambda_{m}>0$ for $\xi<x$ takes the form

$$
\begin{aligned}
T(x, y, z)-T_{i}= & \sum_{m=1}^{\infty} \int_{\xi=-\infty}^{x} q_{w}(\xi) \frac{f_{m}(y, z)}{N_{m}} e^{-\lambda_{m}(x-\xi)} d \xi \int_{\Gamma}\left[f_{m}\left(y^{\prime}, z^{\prime}\right)\right]_{\Gamma^{\prime}} d \Gamma^{\prime} \\
& +\sum_{m=1}^{\infty} \int_{\xi=x}^{\infty} q_{w}(\xi) \frac{g_{m}(y, z)}{N_{m, 1}} e^{\left|\lambda_{m}\right|(x-\xi)} d \xi \int_{\Gamma}\left[g_{m}\left(y^{\prime}, z^{\prime}\right)\right]_{\Gamma^{\prime}} d \Gamma^{\prime}
\end{aligned}
$$

The norm $N_{m}$ is for positive $\lambda_{m}$ and the norm becomes $N_{m, 1}=-$ $N_{m}$ when $\lambda_{m}$ is negative in the definition of $N_{m}$ and they are obtainable from Equation (16b).

\section{Comments and discussion}

The procedure for determination of eigenvalues $\lambda_{m}$ depends on the specific geometry and the functional form of thermophysical properties. For regular geometries with constant thermophysical properties and constant velocity $U$, Equation (22) can be written as

$$
\begin{aligned}
k\left(\frac{\partial^{2} f_{m}(y, z)}{\partial y^{2}}+\frac{\partial^{2} f_{m}(y, z)}{\partial z^{2}}\right) & =-C U \lambda_{m} f_{m}(y, z)-k \lambda_{m}^{2} f_{m}(y, z) \\
& =-\left(C U \lambda_{m}+k \lambda_{m}^{2}\right) f_{m}(y, z) \\
& =k\left(\gamma_{m} / L_{c}\right)^{2} f_{m}(y, z)
\end{aligned}
$$

wherein $f_{m}(y, z)$ satisfies the specified homogeneous boundary conditions. Accordingly, one can determine the function $f_{m}(y, z)$ and the parameter $r_{m}$. Then, eigenvalues are determined from the relation

$$
\lambda_{m}^{2}+\left(\frac{C U}{k}\right) \lambda_{m}-\gamma_{m}^{2} / L_{c}^{2}=0
$$

in Equation (33a) and they are

$$
\begin{aligned}
\lambda_{m} L_{c} & =\frac{1}{2}\left[-\left(\frac{C U L_{c}}{k}\right) \pm \sqrt{\left(\frac{C U L_{c}}{k}\right)^{2}+4 \gamma_{m}^{2}}\right] \\
& =\frac{\mathrm{Pe}}{2}\left[-1 \pm \sqrt{1^{2}+4 \gamma_{m}^{2} / \mathrm{Pe}^{2}}\right]
\end{aligned}
$$


wherein $\mathrm{Pe}=C U L_{c} / k$. This procedure is valid for moving bodies having regular geometries. As illustration, for a parallel plate with prescribed heat flux with insulated walls at $y= \pm H$, this procedure when $L_{c}=H$ produces $f_{m}(r)=\cos \left(\gamma_{m} y / H\right)$ with $\gamma_{m}=m \Pi$. Further details related to this special case are included in the numerical example to appear next. For a moving solid cylinder with radius $r_{0}$ and $L_{c}=r_{0}$, the eigenfunction is $f_{m}(r)=J_{0}\left(\gamma_{m} r / r_{0}\right)$. Then, the parameter $\gamma_{m}$ becomes the mth root of $J_{0}(\gamma)=0$ for a boundary condition of the first kind at $r=r_{0}$. For a boundary condition of the second kind at $r=r_{o^{\prime}}$ the parameter $\gamma_{m}$ becomes the mth root of $J_{1}(\gamma)=0$. It is to be noted that $\Gamma=2 \pi r_{0}$ in Equations (32b) and the norm $N_{m}$ is to be determined from Equations (A.8b) and (A.9b) following integration over the cross-sectional area with $d A=2 \pi r d r$.

When velocity $u$ is position dependent, it is possible to determine the eigenfunctions and eigenvalues in certain applications such as flow between two parallel plates and within circular passages. As discussed earlier in Section 1, an extended Graetz-type solution has been used to accomplish this task in $[7-11,18]$. When the thermophysical properties are position dependent, the method of weighted residuals [12] would directly produce the positive and negative eigenvalues, numerically.

\section{Numerical examples}

Several solutions for moving plates and moving cylinders are given in [4]. These solutions are for constant thermophysical properties and for constant velocity $u=U$. To verify the accuracy of this Green's function solution, consideration is given to the case of moving a plate with thickness $2 H$. This plate is travelling with a uniform velocity $U$ over a region that provides the heat flux on the walls at $y= \pm H$,

$$
q_{w}(x)=\left\{\begin{array}{lll}
0 & \text { when } \quad-\infty<x<0 \\
q_{w} & \text { when } \quad 0 \leq x<\infty
\end{array}\right.
$$

For this moving plate with the walls $2 H$ apart, the eigenfunction is $\cos \left(\gamma_{m} \bar{y}\right)$ with $\bar{y}=y / H$ and $\gamma_{m}=m \pi$. Using the Green's function solution in two-dimensional space $(x, y)$, the temperature solution from Equation (32a), when $x<0$, takes the form

$$
T(x, y)-T_{i}=\sum_{m=0}^{\infty} q_{w} W \frac{\cos (m \pi \bar{y}) \cos (m \pi)}{N_{m, 1} \beta_{m}} e^{\beta_{m} x}
$$

where $\beta_{m}=\left|\lambda_{m}\right|$ when $\lambda_{m}<0$ from Equation (33c) and $W$ is the width of the plate. This summation begins at $m=0$ mainly to include the effect of a constant eigenfunctions. Since $W$ also appears in the definition of $N_{m}$ from Equation (A.8b) where $d A=W d y$, it is appropriate to set $W=1$ in this example. As stated earlier, the subscripts 1 in $N_{m, 1}$ refer to the norms for negative eigenvalues. Using $L_{c}=H$ and the dimensionless parameters $\bar{x}=(x / H) / \mathrm{Pe}$ with $\mathrm{Pe}=\mathrm{CUH} / k$, the Green's function solution, from Equation (35a), takes the form

$$
\frac{k\left[T(x, y)-T_{i}\right]}{q_{w} H}=\frac{1}{\mathrm{Pe}^{2}} \exp \left(\mathrm{Pe}^{2} \bar{x}\right)+\sum_{m=1}^{\infty} \frac{2(-1)^{m} \cos \left(\gamma_{m} \bar{y}\right)}{\hat{\beta}_{m}\left(2 \hat{\beta}_{m} / \mathrm{Pe}^{2}-1\right)} e^{\hat{\beta}_{m} \bar{x}}
$$

The dimensionless form of the eigenvalues, as given in Equation (33c) for $\lambda_{m}<0$, defined as $\hat{\beta}_{m}=\left|\lambda_{m}\right| H \mathrm{Pe}$, is

$$
\hat{\beta}_{m}=\frac{\mathrm{Pe}^{2}}{2}\left[\sqrt{1+4 \gamma_{m}^{2} / \mathrm{Pe}^{2}}+1\right]
$$

and for $\lambda_{m}>0$, defined as $\hat{\lambda}_{m}=\lambda_{m} H \mathrm{Pe}$, is

$$
\hat{\lambda}_{m}=\frac{\mathrm{Pe}^{2}}{2}\left[\sqrt{1+4 \gamma_{m}^{2} / \mathrm{Pe}^{2}}-1\right]
$$

Next, multiply the numerator and the denominator of the coefficient in Equation (35b) by $\lambda_{m}$ and then replace $\hat{\lambda}_{m} \hat{\beta}_{m}$ by their product from Equations (36a) and (36b) to get

$$
\begin{aligned}
\frac{1}{\hat{\beta}_{m}\left(2 \hat{\beta}_{m} / \mathrm{Pe}^{2}-1\right)} & =\frac{\hat{\lambda}_{m}}{\hat{\lambda}_{m} \hat{\beta}_{m}\left(2 \hat{\beta}_{m} / \mathrm{Pe}^{2}-1\right)} \\
& =\frac{\hat{\lambda}_{m}}{\gamma_{m}^{2} \mathrm{Pe}^{2}\left(2 \hat{\beta}_{m} / \mathrm{Pe}^{2}-1\right)}=\frac{\hat{\lambda}_{m}}{\gamma_{m}^{2}\left(2 \hat{\beta}_{m}-\mathrm{Pe}^{2}\right)}
\end{aligned}
$$

Also, it is to be noted from Equations (36a) and (36b) that $\hat{\beta}_{m}$ $\hat{\lambda}_{m}=\mathrm{Pe}^{2}$; therefore, the term $2 \hat{\beta}_{m}-\mathrm{Pe}^{2}$ in this relation can be replaced by $\hat{\beta}_{m}+\lambda_{m}$ to get the related coefficient

$$
\frac{1}{\hat{\beta}_{m}\left(2 \hat{\beta}_{m} / \mathrm{Pe}^{2}-1\right)}=\frac{1}{\gamma_{m}^{2}} \frac{\hat{\lambda}_{m}}{\hat{\lambda}_{m}+\hat{\beta}_{m}}
$$

to be inserted in Equation (35b). Then, the final solution takes the form

$$
\frac{k\left(T-T_{i}\right)}{q_{w} H}=\frac{1}{\mathrm{Pe}^{2}} \exp \left(\operatorname{Pe}^{2} \bar{x}\right)+\sum_{m=1}^{\infty} \frac{2(-1)^{m}}{\gamma_{m}^{2}} \frac{\hat{\lambda}_{m}}{\hat{\lambda}_{m}+\hat{\beta}_{m}} \cos \left(\gamma_{m} \bar{y}\right) \exp \left(\hat{\beta}_{m} \bar{x}\right)
$$

which is identical to the one presented in [4, Equation (34a)].

Also, the temperature solution when $x \geq 0$ is obtainable from Equation (32b) that takes the form

$$
\begin{aligned}
T(x, y)-T_{i}= & \frac{q_{w}}{\operatorname{Pe} C U}+\frac{q_{w} x}{C U H}+\sum_{m=1}^{\infty} q_{w} \frac{\cos (m \pi y / H) \cos (m \pi)}{N_{m} \lambda_{m}} \\
& \times\left(1-e^{-\lambda_{m} x}\right)+\sum_{m=1}^{\infty} q_{w} \frac{\cos (m \pi y / H) \cos (m \pi)}{N_{m, 1} \beta_{m}}
\end{aligned}
$$

The first two terms on the right side of Equation (38a) are the contributions of the first eigenvalues in Equations (32a) and Equation (32b), when $m=0$. In the dimensionless form, Equation (38a) becomes

$$
\begin{aligned}
\frac{k\left[T(x, y)-T_{i}\right]}{q_{w} H}= & \frac{1}{\mathrm{Pe}^{2}}+\bar{x}+\sum_{m=1}^{\infty} \frac{2(-1)^{m} \cos (m \pi \bar{y})}{\hat{\lambda}_{m}\left(2 \hat{\lambda}_{m} / \mathrm{Pe}^{2}+1\right)} \\
& \times\left(1-e^{-\hat{\lambda}_{m} \bar{x}}\right)+\sum_{m=1}^{\infty} \frac{2(-1)^{m} \cos (m \pi \bar{y})}{\hat{\beta}_{m}\left(2 \hat{\beta}_{m} / \mathrm{Pe}^{2}-1\right)}
\end{aligned}
$$

that reduces to take the form

$$
\begin{aligned}
\frac{k\left[T(x, y)-T_{i}\right]}{q_{w} H}= & \frac{1}{\mathrm{Pe}^{2}}+\bar{x}+\sum_{m=1}^{\infty} \frac{2(-1)^{m} \cos (m \pi \bar{y})}{m^{2} \pi^{2}} \\
& -\sum_{m=1}^{\infty} \frac{2(-1)^{m} \cos (m \pi \bar{y})}{\hat{\lambda}_{m}\left(2 \hat{\lambda}_{m} / \mathrm{Pe}^{2}+1\right)} e^{-\hat{\lambda}_{m} \bar{x}}
\end{aligned}
$$

The first summation on the right side of this equation is readily available in Reference [1, Equation (6.79)]; that is

$$
\begin{aligned}
\sum_{m=1}^{\infty} \frac{2(-1)^{m} \cos (m \pi \bar{y})}{m^{2} \pi^{2}} & =\frac{2}{\pi^{2}} \sum_{m=1}^{\infty} \frac{\cos [m \pi(1-\bar{y})]}{m^{2}} \\
& =\frac{1}{2}(1-\bar{y})^{2}-(1-\bar{y})+\frac{1}{3}=\frac{1}{2} \bar{y}^{2}-\frac{1}{6}
\end{aligned}
$$

After substitution for this summation, Equation (38c) reduces to

$$
\begin{aligned}
\frac{k\left(T-T_{i}\right)}{q_{w} H}= & \frac{1}{\operatorname{Pe}^{2}}-\frac{1}{6}+\bar{x}+\frac{1}{2} \bar{y}^{2}-\sum_{m=1}^{\infty} \frac{2(-1)^{m}}{\gamma_{m}^{2}} \frac{\hat{\beta}_{m}}{\hat{\lambda}_{m}+\hat{\beta}_{m}} \\
& \times \cos \left(\gamma_{m} \bar{y}\right) \exp \left(-\hat{\lambda}_{m} \bar{x}\right)
\end{aligned}
$$

which is the same as the solution given in [4, Equation (34b)]. Both Green's function solutions by Equations (37) and (40) confirm the accuracy of this methodology and its future application to more complex problems.

The summations within Equations (37) and (40) are different; however, they are similar and exhibit similar convergence characteristics. When the coordinate $\bar{y}=0$, both series are alternating and they converge with an error of less than 
Table 1. Convergence behavior of the Green's function solution, Equation (40), for Pe $=2$.

\begin{tabular}{lllllll}
\hline$(x / H) / \mathrm{Pe}$ & $N\left(K_{\max }=2.5\right)$ & $k\left(T_{w}-T_{i}\right) / q_{w} H$ & $N\left(K_{\max }=5\right)$ & $k\left(T_{w}-T_{i}\right) / q_{w} H$ & $N\left(K_{\max }=7.5\right)$ & $k\left(T_{w}-T_{i}\right) / q_{w} H$ \\
\hline 0.0001 & 7959 & $0.380106 \underline{\underline{0}}$ & 15,916 & 0.38010659 & 23,874 & 0.38010659 \\
0.0002 & 3980 & $0.3806478 \underline{8}$ & 7959 & 0.38064786 & 11,937 & 0.38064786 \\
0.0005 & 1592 & $0.3821129 \underline{\underline{9}}$ & 3184 & 0.38211293 & 4775 & 0.38211293 \\
0.0010 & 797 & $0.384309 \underline{\underline{8}}$ & 1592 & 0.38430946 & 2388 & 0.38430946 \\
0.0020 & 399 & $0.388266 \underline{\underline{74}}$ & 797 & 0.38826649 & 1194 & 0.38826649 \\
0.0050 & 160 & $0.39858 \underline{\underline{125}}$ & 319 & 0.39858065 & 478 & 0.39858065 \\
0.0100 & 80 & $0.41340 \underline{\underline{9}}$ & 160 & 0.41340373 & 240 & 0.41340373 \\
0.0200 & 41 & $0.43895 \underline{404}$ & 80 & 0.43895206 & 120 & 0.43895206 \\
0.0500 & 17 & $0.50165 \underline{\underline{618}}$ & 33 & 0.50165256 & 49 & 0.50165255 \\
0.1000 & 9 & $0.58657 \underline{440}$ & 17 & $0.5865701 \underline{3}$ & 25 & 0.58657012 \\
\hline
\end{tabular}

$1 / N^{2}$ where $N$ represents the upper limit of the summation, $N=\max (m)$. As the coordinate $\bar{x}$ increases, this error exponentially decreases and becomes equal to zero as $\bar{x} \rightarrow \infty$. However, as $\bar{y}$ increases toward its unit value, the alternative nature of these two series solutions will diminish and the expected error will increase. A remedial procedure to improve convergence, when $\bar{y}=1$, appears later.

As a test of convergence, the numerical values for Equation (40) are found for the number of terms determined from the exponential argument of the last term in the summation; that is

$$
\begin{aligned}
\hat{\lambda}_{m} \bar{x} & =\frac{\mathrm{Pe}^{2}}{2}\left[\sqrt{1+\left(\frac{2 N \pi}{\mathrm{Pe}}\right)^{2}}-1\right] \frac{x}{H} \frac{1}{\mathrm{Pe}} \\
& =\frac{\mathrm{Pe}}{2}\left[\sqrt{1+\left(\frac{2 N \pi}{\mathrm{Pe}}\right)^{2}}-1\right] \frac{x}{H}=K_{\max }
\end{aligned}
$$

The number given using this equation takes advantage of exponential convergence nature of Equation (38b) provided $x$ is not equal to zero and it augments the contribution of $\gamma_{m}^{2}$ $=(m \pi)^{2}$ within the summation. If $K_{\max }=11.5$, then the error in the summation in Equation (38b) will be reduced by $\sim 10^{-5}$ since $\exp (-11.5) \approx 0.0000101$. Then, the value of $N=\max (m)$ is selected by Solving Equation (41) for $N$, that is

$$
N=\text { Ceiling }\left[\frac{K_{\max }}{\pi(x / H)} \sqrt{1+\frac{\operatorname{Pe}(x / H)}{K_{\max }}}\right]
$$

The temperature solutions for $\bar{y}=1$ with designated number of terms, obtained from this equation, are in Table 1 while the inaccurate digits are underlined. In practice, it is not necessary to use $K_{\max }=11.5$, which indeed does give accuracy better than 0.00001 due to the contribution of $1 / \gamma_{m}^{2}$. For that reason temperatures are computed using $K_{\max }=2.5,5$ and 7.5. For the summation given by Equation (40), the number of terms as given tends to be quite conservative. Instead of needing $K_{\max }=11.5$ to get errors less than $10^{-5}$, one can use a much smaller value of $K_{\text {max }}$, even as small as 2.5 . With this value of $K_{\text {max }}$ less than 20 terms are needed for $\bar{x}$ greater than 0.05 . In fact, less than 10 terms are needed for $\bar{x}$ greater than 0.1 . This convergence criterion shows that the required number of terms depends upon the size of $\bar{x}$ and can be as small as 1 and up to the 1000s.

The above method of solution is particularly effective for $\bar{x}>0.005$. When the axial coordinate $\bar{x}$ has small values, another method is needed which utilizes the nature of the solution when $\bar{x} \ll 1$. To show the convergence behaviors of Equations (37) and (40), they can be written as

$$
\begin{aligned}
\frac{k\left(T-T_{i}\right)}{q_{w} H}= & \frac{1}{\operatorname{Pe}^{2}} \exp \left(\operatorname{Pe}^{2} \bar{x}\right)+\sum_{m=1}^{\infty} \\
& \times \frac{(-1)^{m}}{\gamma_{m}^{2}}\left(1-\frac{\beta_{m}-\lambda_{m}}{\beta_{m}+\lambda_{m}}\right) \cos \left(\gamma_{m} \bar{y}\right) \exp \left(\beta_{m} \bar{x}\right)
\end{aligned}
$$

when $\bar{x} \leq 0$, and

$$
\begin{aligned}
\frac{k\left(T-T_{i}\right)}{q_{w} H}= & \frac{1}{\mathrm{Pe}^{2}}-\frac{1}{6}+\bar{x}+\frac{1}{2} \bar{y}^{2}-\sum_{m=1}^{\infty} \\
& \times \frac{(-1)^{m}}{\gamma_{m}^{2}}\left(1+\frac{\beta_{m}-\lambda_{m}}{\beta_{m}+\lambda_{m}}\right) \cos \left(\gamma_{m} \bar{y}\right) \exp \left(-\lambda_{m} \bar{x}\right)
\end{aligned}
$$

when $\bar{x} \geq 0$. There are two summations in each of these equations; they are

$$
\Psi_{1}=\sum_{m=1}^{\infty} \frac{(-1)^{m}}{\gamma_{m}^{2}} \cos \left(\gamma_{m} \bar{y}\right) \exp \left(-\left|\lambda_{m}\right||\bar{x}|\right)
$$

and

$$
\Psi_{2}=\sum_{m=1}^{\infty} \frac{(-1)^{m}}{\gamma_{m}^{2}} \cos \left(\gamma_{m} \bar{y}\right)\left(\frac{\beta_{m}-\lambda_{m}}{\beta_{m}+\lambda_{m}}\right) \exp \left(-\left|\lambda_{m}\right||\bar{x}|\right)
$$

wherein $\beta_{m}$ replaces $\left|\lambda_{m}\right|$ when $\bar{x}<0$. A Fourier series analysis shows that, at $\bar{x}=0$, the function $\Psi_{1}$ has an exact value

$$
\Psi_{1}=\frac{1}{12}\left(3 \bar{y}^{2}-1\right)
$$

and it provides $\Psi_{1}=-1 / 12$ at $\bar{y}=0$ and $\Psi_{1}=1 / 6$ at $\bar{y}=1$. The main task is to further elaborate on the general convergence behaviors of these two series for $N=\max (m)$ terms in each series. Combining parameters $\hat{\beta}_{m}$ and $\hat{\lambda}_{m}$ from Equations (36a) and $(36 \mathrm{~b})$ produces the parameters $\hat{\beta}_{m}-\hat{\lambda}_{m}=\mathrm{Pe}^{2}$ and $\hat{\beta}_{m}+\lambda_{m}=$ $\mathrm{Pe}^{2}\left[1+(2 m \pi / \mathrm{Pe})^{2}\right]^{1 / 2}$. Additionally, when the quantity $2 N \Pi /$ $\mathrm{Pe} \gg 1$, one can get a single approximate value of $\max \left(\left|\lambda_{m}\right|\right)$ $\approx N \pi$ Pe from Equations (36a) and (36b), and for the worst-case scenario, when $\bar{y}=1$, the error for the functions $\Psi_{1}$ is

$$
\operatorname{Error}\left(\Psi_{1}\right)<\frac{1}{\pi^{2}} \exp [-(N+1) \pi \operatorname{Pe}|\bar{x}|] \sum_{m=N+1}^{\infty} \frac{1}{m^{2}}
$$

and for function $\Psi_{2}$ is

$$
\operatorname{Error}\left(\Psi_{2}\right)<\frac{\operatorname{Pe}}{2 \pi^{3}} \exp [-(N+1) \pi \operatorname{Pe}|\bar{x}|] \sum_{m=N+1}^{\infty} \frac{1}{m^{3}}
$$

To accurately estimate the above summation, consider $m$ to be a continuous function $f(m)=1 / m^{v}$. Next, the function $f(m)$ $\Delta m=\Delta m / m^{v}$ represents an infinitesimal area and the total discretized area below $f(m)$ line is 
Table 2. Temperature at $x=0$ and $y=H$ for various Pe numbers and numbers of terms.

\begin{tabular}{llllll}
\hline$N$ & $\mathrm{Pe}=1$ & $\mathrm{Pe}=2$ & $\mathrm{Pe}=5$ & $\mathrm{Pe}=10$ & $\mathrm{Pe}=20$ \\
\hline 10 & 1.147490518 & 0.379477126 & 0.128136988 & 0.063659935 & 0.031799074 \\
20 & 1.147490522 & 0.379477158 & 0.128137472 & 0.063663770 & 0.031828708 \\
50 & 1.147490522 & 0.379477160 & 0.128137507 & 0.063664049 & 0.031830926 \\
100 & 1.147490522 & 0.379477160 & 0.128137508 & 0.063664057 & 0.031830985 \\
200 & 1.147490522 & 0.379477160 & 0.128137508 & 0.063664057 & 0.031830988 \\
500 & 1.147490522 & 0.379477160 & 0.128137508 & 0.063664057 & 0.031830989 \\
1000 & 1.147490522 & 0.379477160 & 0.128137508 & 0.063664057 & 0.031830989 \\
\hline
\end{tabular}

$$
\lim _{\Delta m \rightarrow 0} \sum_{m=N_{1}}^{\infty} \frac{\Delta m}{m^{v}}=\int_{m=N_{2}}^{\infty} \frac{d m}{m^{v}}=\frac{1}{(v-1) N_{2}^{v-1}}
$$

When $\Delta m=1$ and $N_{1}=N+1$, the right side of this equation provides an estimate of the needed summation when $N_{2}=N_{1}-\Delta m / 2 \approx N$; that becomes

$$
\sum_{m=N+1}^{\infty} \frac{1}{m^{v}} \approx \frac{1}{(v-1) N^{v-1}}
$$

Then, the expected error after replacing the summations in Equations (45a) and (45b) is

$$
\operatorname{Error}\left(\Psi_{1}\right)<\frac{1}{\pi^{2} N} \exp [-(N+1) \pi \operatorname{Pe}|\bar{x}|]
$$

and for function $\Psi_{2}$ is

$$
\operatorname{Error}\left(\Psi_{2}\right)<\frac{\operatorname{Pe}}{4 \pi^{3} N^{2}} \exp [-(N+1) \pi \operatorname{Pe}|\bar{x}|] \sum_{m=N+1}^{\infty} \frac{1}{m^{3}}
$$

This indicates that the expected error of $\exp \left(-K_{\max }\right)$, as given by Equation (42), should be reduced by a factor of $1 /\left(N \pi^{2}\right)$.

It is also possible to enhance the accuracy of the wall temperature values, using a moderately high value of $N$, by adding and subtracting the same function in different functional forms. Accordingly, Equation (40) for determination of the surface temperature at $\bar{y}=0$ takes the form

$$
\begin{aligned}
\frac{k\left(T-T_{i}\right)}{q_{w} H} & =\frac{1}{\mathrm{Pe}^{2}}-\frac{1}{6}+\bar{x}+\frac{1}{2}-e^{\mathrm{Pe}^{2} \bar{x} / 2} \\
& \times\left\{\frac{1}{\pi^{2}} L i_{2}\left(e^{-\pi \overline{\mathrm{x} P e}}\right)+\sum_{m=1}^{\infty} \frac{1}{(m \pi)^{2}}\left(e^{-\mathrm{Pe}^{2} \bar{x} \sqrt{1+(2 m \pi / \mathrm{Pe})^{2}} / 2}-e^{-m \pi \overline{\mathrm{xPe}}}\right)\right. \\
& \left.+\frac{\mathrm{Pe}}{2} \frac{1}{\pi^{3}} L i_{3}\left(e^{-\pi \overline{\mathrm{x} P e}}\right)+\frac{\mathrm{Pe}}{2} \sum_{m=1}^{\infty} \frac{1}{(m \pi)^{3}}\left[\frac{e^{-\mathrm{Pe}^{2} \bar{x} \sqrt{1+(2 m \pi / \mathrm{Pe})^{2}} / 2}}{\sqrt{1+\mathrm{Pe}^{2} /(2 m \pi)^{2}}}-e^{-m \pi \overline{\mathrm{PPe}}}\right]\right\}
\end{aligned}
$$

Repeating this algebraic process for Equation (37) produces the similar summation terms and it leads to the relation

$$
\begin{aligned}
\frac{k\left(T-T_{i}\right)}{q_{w} H} & =\frac{1}{\mathrm{Pe}^{2}} e^{-\mathrm{Pe}^{2}|\bar{x}|}+e^{-\mathrm{Pe}^{2}|\bar{x}| / 2} \\
& \times\left\{\frac{1}{\pi^{2}} L i_{2}\left(e^{-\pi|\bar{x}| \mathrm{Pe}}\right)+\sum_{m=1}^{\infty} \frac{1}{(m \pi)^{2}}\left(e^{-\mathrm{Pe}^{2}|\bar{x}| \sqrt{1+(2 m \pi / \mathrm{Pe})^{2}} / 2}-e^{-m \pi|\bar{x}| \mathrm{Pe}}\right)\right. \\
& \left.-\frac{\mathrm{Pe}}{2} \frac{1}{\pi^{3}} L i_{3}\left(e^{-\pi|\bar{x}| \mathrm{Pe}}\right)-\frac{\mathrm{Pe}}{2} \sum_{m=1}^{\infty} \frac{1}{(m \pi)^{3}}\left[\frac{e^{-\mathrm{Pe}^{2}|\bar{x}| \sqrt{1+(2 m \pi / \mathrm{Pe})^{2}} / 2}}{\sqrt{1+\mathrm{Pe}^{2} /(2 m \pi)^{2}}}-e^{-m \pi|\bar{x}| \mathrm{Pe}}\right]\right\}
\end{aligned}
$$

The functions $L i_{1}$ and $L i_{2}$ in Equations (48a) and (48b) are standard classical function; e.g., called PolyLog function in the standard symbolic software Mathematica [24]. By definition, the PolyLog function describes the summation below

$$
L_{v}\left(e^{-\pi \bar{x}}\right)=\sum_{m=1}^{\infty} \frac{\left(e^{-\pi \bar{x}}\right)^{v}}{m^{v}}
$$

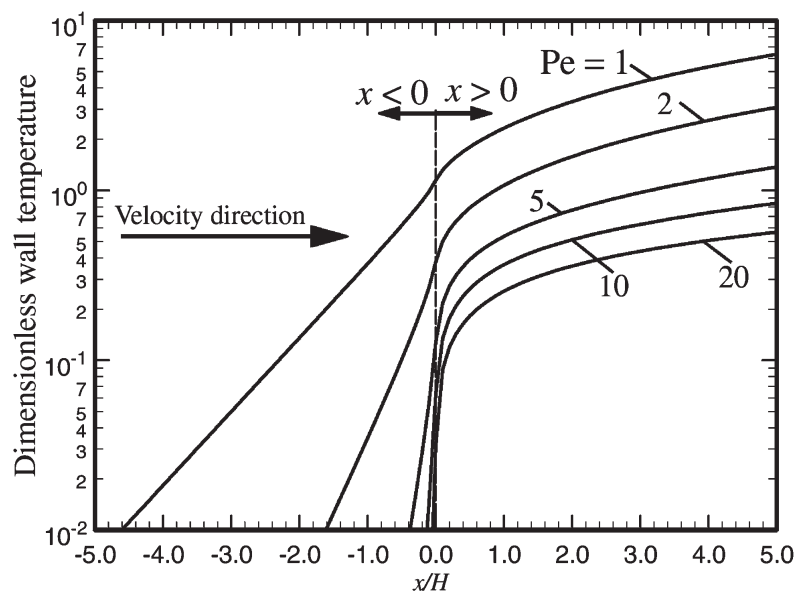

Figure 2. Dimensionless surface temperature $k\left(T_{w}-T_{i}\right) /\left(q_{w} H\right)$ of a moving solid as a function of $x / H$ for different dimensionless velocities $\mathrm{Pe}=U H / a$, when $q_{w}=$ constant at $x>0$.

The convergence of Equation (48a) at $\bar{x}=0$ is numerically demonstrated in Table 2. The error of the summation when $\mathrm{Pe} /(\Pi N) \ll 1$ is deterministic for each of the summations in Equation (48a) which are the same as those in Equation (48b). The error in the first summation becomes

$$
\sum_{m=N+1}^{\infty} \frac{\bar{x}}{8}\left(\frac{\mathrm{Pe}}{m \pi}\right)^{3} \exp \left[-\left(\frac{\bar{x}}{2}\right)(m \pi \mathrm{Pe})\left(2-\frac{\mathrm{Pe}}{m \pi}\right)\right]
$$

This error vanishes when $\bar{x}=0$ and, as $\bar{x}$ increases, its contribution remains negligibly small. The error for the second summation in Equation (48a) is

$$
-\sum_{m=N+1}^{\infty}\left[\frac{\bar{x}}{16}\left(\frac{\mathrm{Pe}}{m \pi}\right)^{4}+\frac{1}{16}\left(\frac{\mathrm{Pe}}{m \pi}\right)^{5}\right] \exp \left[-\left(\frac{\bar{x}}{2}\right)(m \pi \mathrm{Pe})\left(2-\frac{\mathrm{Pe}}{m \pi}\right)\right]
$$

The numerical values of these errors can be estimated using Equation (46b). The results show significant error reductions in comparison to those from Equations (47a) and (47b), especially when $\bar{x}$ is small. Note that the maximum value of a function $\bar{x} \exp (-a \bar{x})$ is equal to $\exp (-1) / a<1$ while $a>1$ in these relations. As a test case, it is to be noted from data in Table 2 that $N=50$ yields a temperature of 0.379477160 with 9 accurate decimal places for $\mathrm{Pe}=2$. This clearly agrees with the estimated error, using Equation $(46 \mathrm{~b})$, to be $(1 / 16)(\mathrm{Pe} / \pi)^{5} /\left(4 \mathrm{~N}^{4}\right)=2.61 \times 10^{10}$. However, now instead of needing 50,000 terms, only 50 terms are needed and this is a very satisfactory feature.

Figure 2 shows the behavior of dimensionless wall temperature $k\left(T_{w}-T_{i}\right) /\left(q_{w} H\right)$ as a function of axial coordinate $x / H$, for different Peclet numbers $\mathrm{Pe}=U H / a$. The plotted values show a rapid rise in the wall temperature as Pe reduces to below 2. This graph includes information directly acquired from Equations (35a) and (38a). Using an energy balance, one can show that the slope of these lines should approach $1 / \mathrm{Pe}$ at large values of $x / H$. 
In the presence of a discrete heat flux, of a constant value in the region between $x=0$ and $x=\Delta x$, and zero heat flux elsewhere, Equation (31) takes the form

$$
\begin{aligned}
T(x, y, z)-T_{i}= & \frac{1}{C(\vec{r})} \int_{\xi=0}^{\Delta x} d \xi \int_{\Gamma}[q G]_{\Gamma^{\prime}} d \Gamma^{\prime} \\
= & \frac{1}{C(\vec{r})} \int_{\xi=0}^{\infty} d \xi \int_{\Gamma}[q G]_{\Gamma^{\prime}} d \Gamma^{\prime}-\frac{1}{C(\vec{r})} \\
& \times \int_{\xi=\Delta x}^{\infty} d \xi \int_{\Gamma}[q G]_{\Gamma^{\prime}} d \Gamma^{\prime}
\end{aligned}
$$

This simplifies the computational procedure and Figure 3 shows the dimensionless wall temperature $k\left(T_{w}-T_{i}\right) /\left(q_{w w} H\right)$ as a function of axial coordinate $x / H$, for different Peclet numbers $\mathrm{Pe}=U H / \alpha$ and for heating limited to a small area. The heated zone is symmetric and it has a size $\Delta x / H=1$. It is to be noted that, when $\mathrm{Pe}=1$, the temperature rapidly approaches its limiting value $(\Delta x / H) / \mathrm{Pe}=1 / \mathrm{Pe}$. This asymptotic behavior is realized at larger values of $x / H$ as Pe increases.

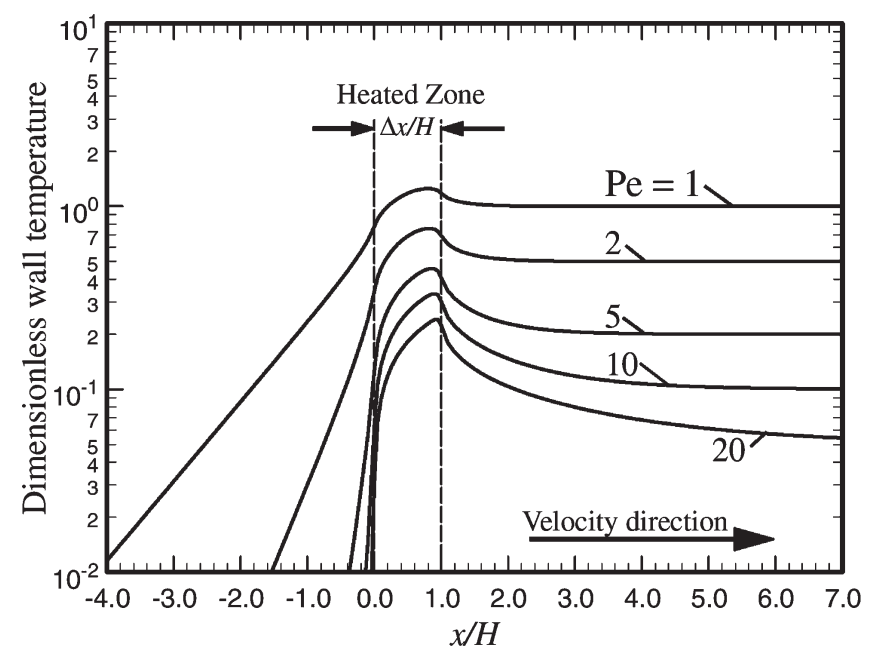

Figure 3. Dimensionless surface temperature $k\left(T_{w}-T_{i}\right) /\left(q_{w} H\right)$ for a moving solid as a function of $x / H$ for different dimensionless velocities $\mathrm{Pe}=U H / \alpha$, when $q_{w}=$ constant at $0<x / H<1$.

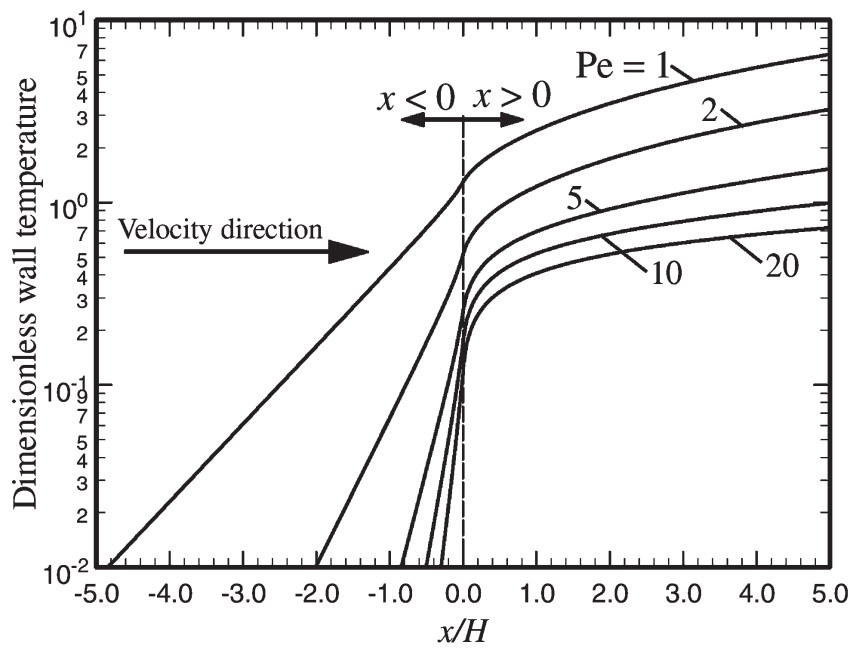

Figure 4. Dimensionless surface temperature $k\left(T_{w}-T_{i}\right) /\left(q_{w} H\right)$ of a moving fluid as a function of $x / H$ for different dimensionless velocities $\mathrm{Pe}=U H / \alpha$, when $q_{w}=$ constant at $x>0$.

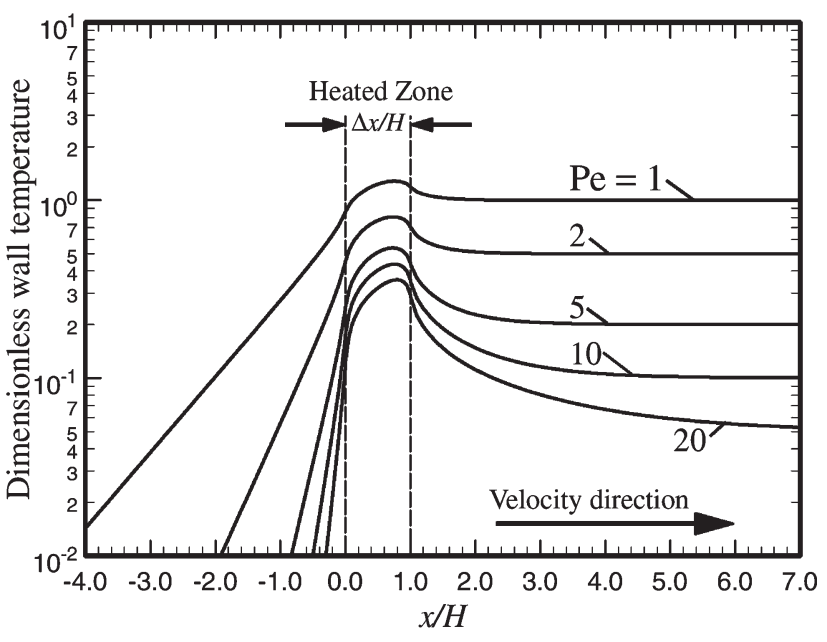

Figure 5. Dimensionless surface temperature $k\left(T_{w}-T_{j}\right) /\left(q_{w} H\right)$ for a moving fluid as a function of $x / H$ for different dimensionless velocities $\mathrm{Pe}=U H / \alpha$, when $q_{w}=$ constant at $0<x / H<1$.

For the next example of the application of this methodology, the moving solid between the two parallel plates is replaced by a laminar fluid flow with a velocity profile

$$
u(y)=\frac{3}{2} U\left[1-\left(\frac{y}{H}\right)^{2}\right]
$$

where $U$ is the average velocity. The method of determination of eigenvalues using an extended Graetz-type solution is presented in [18] and an alternative methodology is presented in [12]. Figure 4 shows the dimensionless wall temperature as a function $x / H$, for different $\mathrm{Pe}=U H / \alpha$ values, for heating over the region $x>0$. The data in Figure 4 behave similar to those in Figure 2 except they have higher values near the thermal entrance location. To show this effect for a finite heat source at the wall, Figure 5 is prepared similar to Figure 3 except it is for a moving fluid. The entries in Figure 5 behave similar to those plotted in Figure 3 except they have higher values near the thermal entrance location.

\section{Conclusion}

The study presented herein indicates that the contribution of axial conduction becomes significant when the velocity in a moving body is relatively small. This effect causes significant upstream temperature penetration when the Peclet number is small. The data for the limiting case of slug flow between two parallel plates indicate this behavior. Indeed, for flow through a clear passage, this upstream temperature penetration significantly increases.

The steady-state Green's function solution, in Equation (8), provides useful information for application to flow in microchannels and through porous passages. In addition to the thermal effect of the wall conditions, it accommodates the contribution of frictional heating. This solution methodology also has useful applications in electronic cooling. With appropriate eigenfunctions, one can determine the thermal effects of discretely placed energy sources along the walls.

\section{Appendix A.}

When function $\theta$ is the temperature solution in a moving body with constant cross-section, this solution, in the presence of homogeneous boundary conditions, can be specified as 


$$
\theta(x, y, z)=\sum_{m=1}^{\infty} B_{m} f_{m}(y, z) e^{-\lambda_{m} x}
$$

This solution must satisfy the homogeneous form of the diffusion equation, Equation (1) with $S=0$. Then, the function $f_{m}(y, z)$ within this series solution for any index $m$ must satisfy the relations

$$
\nabla \cdot\left(k \nabla f_{m}(y, z)+\lambda_{m}\left(\lambda_{m} k+C u\right) f_{m}(y, z)-\omega^{2} f_{m}(y, z)=0\right.
$$

Also, when $f_{m}(y, z)$ is replaced by $g_{n}(y, z)$ and $\lambda_{m}$ replaced by $\beta_{n^{\prime}}$ Equation (A.2a) becomes

$$
\nabla \cdot\left(k \nabla g_{n}(y, z)+\beta_{n}\left(\beta_{n} k+C u\right) g_{n}(y, z)-\omega^{2} g_{n}(y, z)=0\right.
$$

It is to be noted that $f_{m}(y, z)=g_{n}(y, z)$ when $m=n$ and $\lambda_{m}=\beta_{n}$. After multiplying Equation (A.2a) by $g_{n}(y, z)$ and Equation (A.2b) by $-f_{m}(y, z)$ and, after adding the resulting relations, it yields,

$$
\begin{array}{r}
\nabla \cdot\left(k \nabla f_{m}(y, z) g_{n}(y, z)-\nabla \cdot\left(k \nabla g_{n}(y, z) f_{m}(y, z)\right.\right. \\
=-\lambda_{m}\left(\lambda_{m} k+C u\right) f_{m}(y, z) g_{n}(y, z) \\
+\beta_{n}\left(\beta_{n} k+C u\right) f_{m}(y, z) g_{n}(y, z)
\end{array}
$$

Adding and subtracting the quantity

$$
\lambda_{m} \beta_{n} k f_{m}(y, z) g_{n}(y, z)
$$

to the right side of this Equation (A.3) produces

$$
\begin{aligned}
\nabla & \cdot\left(k \nabla f_{m}(y, z) g_{n}(y, z)-\nabla \cdot\left(k \nabla g_{n}(y, z) f_{m}(y, z)\right.\right. \\
= & -\lambda_{m}\left[\left(\lambda_{m}+\beta_{n}\right) k+C u\right] f_{m}(y, z) g_{n}(y, z) \\
& +\beta_{n}\left[\left(\lambda_{m}+\beta_{n}\right) k+C u\right] f_{m}(y, z) g_{n}(y, z)
\end{aligned}
$$

This equation is to be integrated over the area normal to the $x$-direction and after using the procedure in Equation (10.14) within Beck et al. [1], one gets

$$
\begin{aligned}
\int_{A}\left(\lambda_{m}-\beta_{n}\right)\left[\left(\lambda_{m}+\beta_{n}\right) k+C(y, z) u(y, z)\right] f_{m} g_{n} d A \\
=-\int_{A}\left\{\nabla \cdot\left[k \nabla f_{m}(y, z)\right] g_{n}(y, z)\right\} d y d k \\
\quad+\int_{A}\left\{\nabla \cdot\left[k \nabla g_{n}(y, z)\right] f_{n}(y, z)\right\} d y d z \\
=\int_{\Gamma} k\left\{f_{m}(y, z)\left[\frac{\partial g_{n}(y, z)}{\partial n}\right]-g_{n}(y, z)\left[\frac{\partial f_{m}(y, z)}{\partial n}\right]\right\}_{\Gamma} d \Gamma
\end{aligned}
$$

The right side of this equation unconditionally vanishes for boundary conditions of first, second, or third kind and this makes

$$
\left(\lambda_{m}-\beta_{n}\right) \int_{A}\left[\left(\lambda_{m}+\beta_{n}\right) k+C u\right] f_{m}(y, z) g_{n}(y, z) d y d z=0
$$

This represents the orthogonality condition; and there are three possible cases: The first case appears when the product $\beta_{b} \lambda_{m}<0$ and for this case

$$
\int_{A}\left[\left(\lambda_{m}+\beta_{n}\right) k+C u\right] f_{m}(y, z) g_{n}(y, z) d y d z=0
$$

unconditionally. The second case is when $\beta_{n} \lambda_{m}>0$ and $\beta_{n}<0$. For this case, one can replace $\lambda_{m}$ with $\beta_{m}$ in Equation (A.6) to get the orthogonality condition

$$
\int_{A}\left[\left(\beta_{m}+\beta_{n}\right) k+C u\right] f_{m} g_{n} d A=\left\{\begin{array}{lll}
0 & \text { when } & m \neq n \\
N_{m} & \text { when } & m=n
\end{array}\right.
$$

and this makes

$$
N_{m}=\int_{A}\left(2 \beta_{m} k+C u\right)\left[f_{m}(y, z)\right]^{2} d A
$$

since $\lambda_{m}$ can be positive or negative. Finally, case 3 is when $\beta_{n}$ $\lambda_{m}>0$ and $\lambda_{m}>0$. This case, replacing $\beta_{n}$ with $\lambda_{n}$ in Equation (A.6), modifies Equations (A.8a) and (A.8b) to read

$$
\int_{A}\left[\left(\lambda_{m}+\lambda_{n}\right) k+C u\right] f_{m} g_{n} d A=\left\{\begin{array}{lll}
0 & \text { when } m \neq n \\
N_{m} & \text { when } m=n
\end{array}\right.
$$

and this makes

$$
N_{m}=\int_{A}\left(2 \lambda_{m} k+C u\right)\left[f_{m}(y, z)\right]^{2} d A .
$$

\section{References}

[1] J. V. Beck, K. D. Cole, A. Haji-Sheikh, and B. Litkouhi, Heat Conduction Using Green's Functions, Hemisphere, Washington, DC (1992).

[2] H. Nagasue, Steady-state heat transfer with axial conduction in laminar flow in a circular tube with a specified temperature or heat flux wall, Int. J. Heat Mass Transfer 24 (11) (1981), pp. 1823-1832.

[3] A. Haji-Sheikh, D. E. Amos, and J. V. Beck, Axial heat conduction of heat through a moving fluid in a semi-infinite region, Int. J. Heat Mass Transfer 51 (19-20) (2008), pp. 4651-4658.

[4] A. Haji-Sheikh, D. E. Amos, and J. V. Beck, Temperature field in a moving semi-infinite region with a prescribed wall heat flux, Int. J. Heat Mass Transfer 51 (7-8) (2009), pp. 2092-2101.

[5] I. Tiselj, G. Hetsroni, B. Mavko, A. Mosyak, E. Pogrebnyak, and Z. Segal, Effect of axial conduction on the heat transfer in micro-channels, Int. J. Heat Mass Transfer 47 (12-13) (2004), pp. 2551-2565.

[6] A. Haji-Sheikh, W.J. Minkowycz, and E.M. Sparrow, Green's function solution of temperature field for flow in porous passages, Int. J. Heat Mass Transfer 47 (22) (2004), pp. 4685-4695.

[7] J. Lahjomri and A. Oubarra, Analytical solution of the Graetz problem with axial conduction, ASME J. Heat Transfer 121 (4) (1999), pp. 1078-1083.

[8] M.L. Michelsen and J. Villadsen, The Graetz problem with axial heat conduction, Int. J. Heat Mass Transfer 17 (11) (1974), pp. 1391-1402.

[9] A. Haji-Sheikh, J. V. Beck, and D. E. Amos, Axial heat conduction effects in the entrance region of parallel plate ducts, Int. J. Heat Mass Transfer $\mathbf{1 0}$ (25-26) (2008), pp. 5811-5822.

[10] A. Haji-Sheikh, J. V. Beck, and D. E. Amos, Axial heat conduction effects in the entrance region of circular ducts, Heat Mass Transfer 45 (3) (2009), pp. 331-341.

[11] A. Sharma and S. Chakraborty, Semi-analytical solution of the extended Graetz problem for combined electroosmotically and pressure-driven microchannel flows with step-change in wall temperature, Int. J. Heat Mass Transfer 51 (19-29) (2008), pp. 4875-4885.

[12] A. Haji-Sheikh, Determination of heat transfer in ducts with axial conduction by variational calculus, ASME J. Heat Transfer 131 (9) (2009) 09-1702-(1-11).

[13] M. Faghri and J. R. Welty, Analysis of transfer, including axial fluid conduction for laminar tube flow with arbitrary circumferential wall heat flux variation, Int. J. Heat Mass Transfer 21 (3) (1978), pp. 317-323.

[14] R. G. Najjar and C. Laohakul, An approximate solution to the Graetz problem with axial conduction and prescribed wall heat flux, Int. Commun. Heat Mass Transfer 13 (3) (1986), pp. 315-324.

[15] D. A. Nield, A. V. Kuznetsov, and M. Xiong, Thermally developing forced convection in a porous medium: parallel plate channel with walls at uniform temperature, with axial conduction and viscous dissipation effects, Int. J. Heat Mass Transfer 46 (4) (2003), pp. 643-651.

[16] A. V. Kuznetsov, D. A. Nield, and M. Xiong, Thermally developing forced convection in a porous medium: circular ducts with walls at constant temperature, with longitudinal conduction and viscous dissipation effects, Transp. Porous Med. 53 (3) (2003), pp. 331-345.

[17] S. Tada and K. Ichimiya, Analysis of laminar dissipative flow and heat transfer in porous saturated circular tube with constant wall heat flux, Int. J. Heat Mass Transfer 50 (11-12) (2007), pp. 2406-2413.

[18] W. J. Minkowycz and A. Haji-Sheikh, Heat transfer in parallel-plate and circular porous passages with axial conduction, Int. J. Heat Mass Transfer 49 (13-14) (2006), pp. 2381-2390.

[19] W. J. Minkowycz and A. Haji-Sheikh, Asymptotic behaviors of heat transfer in porous passages with axial conduction, Int. J. Heat Mass Transfer 52 (13-14) (2009), pp. 3101-3108.

[20] D. H. Y. Yen and J. V. Beck, Green's function and three-dimensional steady-state heat-conduction problems in a two-layered composite, $J$. Eng. Math. 49 (3) (2004), pp. 305-319.

[21] K. D. Cole, Computer programs for temperature in fins and slab bodies with the method of Green's functions, Comput. Appl. Eng. Educ. 12 (3) (2004), pp. 189-197.

[22] K. D. Cole, Steady-periodic Green's functions and thermal-measurement applications in rectangular coordinates, ASME J. Heat Transfer 128 (7) (2006), pp. 709-716.

[23] K. D. Cole, Steady-periodic heating in parallel-plate microchannel flow with participating walls, Int. J. Heat Mass Transfer 53 (5-6) (2009), pp. 870-878.

[24] S. Wolfram, The Mathematica Book (5th ed.), Cambridge University Press, Cambridge (2005). 